\title{
Diversidad de mamíferos y participación local en la conservación en el Gran Chaco Boliviano
}

Introduction: Local participation, be it passive or active, has had variable degrees of success in long-term conservation processes. We describe an alliance in the Bolivian Gran Chaco between indigenous peopleseeking land and resource rights, and improved livelihoods—and a conservation organization—pursuing biodiversity and wildlife conservation-that successfully proposed, and subsequently managed, a vast national park comprising one of the best preserved and most extensive areas of tropical dry forest in the world. We explain the geographical, political / social and biological contexts (relative to mammals) of this partnership in order to encourage collaboration elsewhere in Latin America.

The Gran Chaco's varied habitats support a diversity of unique species, but like other tropical dry forests, it is suffering severe pressure from the expansion of industrial agriculture and hunting. Created in 1996, the Kaa-lya National Park covers $34,000 \mathrm{~km}^{2}$ in Bolivia. Extensive and participatory ecological and socioeconomic studies, combined with an intense consultation process, resulted in a zonification that provides areas for agriculture and resource exploitation by private land-owners and by indigenous peoples-Ayoreode, Chiquitano, and Isoseño-Guaraní-neighboring the park. Subsequent participatory research and training in the park and on neighboring indigenous communal lands established a cadre of indigenous "parabiologists."

Methodology: Research by joint parabiologist-biologist teams on mammals included efforts to document the status of the Chacoan guanaco Lama guanicoe; research on the ecology of the jaguar Panthera onca and its prey; conservation and use of landscape species (including the endemic Chacoan peccary Catagonus wagneri); resolution of conflicts between humans and wildlife; a fire ecology study to understand the structural changes in the landscape; and a binational Bolivia-Paraguay effort to survey mammals.

Results: Isoseño-Guaraní communities, in response to the findings on guanacos and Chacoan peccaries, proposed and adopted a ban on hunting these two endangered species. With respect to game species, hundreds of hunters participated in a multi-year self-monitoring program. After analyzing the data, the communities adopted temporary hunting bans on lowland tapirs Tapirus terrestris and white-lipped peccaries Tayassu pecari, management plans for the sustainable commercial utilization (skins) of collared peccary Pecari tajacu, and defined communal reserves with no hunting. The fire ecology study found that traditional fire management by indigenous communities maintained the most diverse savanna habitats.

Discussion and conclusions: A successful partnership must recognize that the interests or general objectives of the actors may vary, explicitly identify common ground, and collaborate to promote objectives that coincide or that complement each other. Through participatory research and formal training activities (including an 8 month, 13 module certification program), the parabiologists developed their own research projects, analyzed data they had collected, presented the results to their communities and wider audiences, and worked with the communities to manage and conserve wildlife resources and communal lands. Several have become leaders of their communities and directed local development projects. The benefits of participatory conservation and alliances between indigenous peoples and conservation organizations therefore far exceed the immediate outcomes of databases and publications on wildlife, providing technical and human resources as well as personal trust and institutional collaboration mechanisms as a base for long-term landscape conservation. Nevertheless continuity depends on action and financial support, and

\footnotetext{
${ }^{1}$ Fundación Gente y Conservación, Santa Cruz de la Sierra, Bolivia. E-mail erika.cuellar71@gmail.com (EC) ${ }^{2}$ Department of Geography, 3141 Turlington Hall, P.O. Box 117315, Gainsville, Forida 32611. Estados Unidos de Norte America. E-mail andrew.noss@yahoo.com (AJN)

*Corresponding author
} 
partners must strive to develop new allies that include in the Kaa-lya case local and national government entities, private landowners, and business such as the natural gas industry.

Key words: Bolivia, Gran Chaco, indigenous people, tropical dry forest, parabiologist.

La participación local en los procesos de conservación, sea esta pasiva o activa, ha tenido resultados de éxito variables. Está claro que una alianza exitosa entre actores depende principalmente en reconocer que los intereses u objetivos generales pueden variar. A manera de ilustrar este enfoque de alianzas entre actores locales y conservacionistas presentamos la experiencia en una parte del Gran Chaco boliviano. Explicamos los contextos geográfico, político/social, y biológico (referido a mamíferos) de esta alianza de trabajo entre un pueblo indígena y un grupo conservacionista que proponía la protección del área de bosque seco tropical mejor conservado y más extenso del mundo.

La estructura de trabajo dentro del contexto del estudio de los mamíferos incluyó esfuerzos para documentar la situación del guanaco chaqueño Lama guanicoe; investigación sobre ecología del jaguar Panthera onca y sus presas; conservación y uso de especies paisaje (incluyendo el endémico pecarí chaqueño Catagonus wagneri); resolución de conflictos entre humanos y vida silvestre; esfuerzos para entender los cambios estructurales en el paisaje realizando estudios en ecología del fuego; y finalmente el desarrollo de esfuerzos binacionales (Bolivia-Paraguay) para el relevamiento de mamíferos y el entrenamiento de parabiólogos.

Palabras clave: Bolivia, bosque tropical seco, Gran Chaco, parabiólogo, pueblo indígena.

La participación local en los procesos de conservación y desarrollo se ha caracterizado Introducción por ser pasiva o llevada a una "auto-movilización" de los actores locales (Pimbert y Pretty 1994). Está claro que en los procesos de conservación se han intentado muchas formas de alianza entre diferentes actores locales; dichos intentos han tenido resultados de éxito variables. Aparentemente, una alianza exitosa entre actores depende principalmente en reconocer que los intereses u objetivos generales pueden variar. Sin embargo, se pueden encontrar áreas de solapamiento para colaborar entre diferentes actores y así generar ciertos beneficios comunes (Redford y Fearn 2007). A manera de ilustrar este enfoque de alianzas entre actores locales y conservacionistas (Castillo et al. 2006), se resume a continuación los hechos en tres grandes temas (contexto geográfico, contexto político/ social, contexto biológico) de la experiencia de trabajo entre un pueblo indígena cuyo objetivo era consolidar su territorio ancestral, protegiendo la fuente de sus recursos de caza y pesca, y un grupo conservacionista que proponía la protección del área de bosque seco tropical mejor conservado y más extenso del mundo.

Los tres contextos mencionados se pueden resumir en una combinación de factores que describiremos en este trabajo. Sugerimos tomar en cuenta esta experiencia dado que la misma podría ser adaptada y aplicada en diferentes países de América latina, para lograr objetivos de integración de actores locales y conservación de los recursos naturales a largo plazo. 
Contexto geográfico. Los bosques tropicales secos forman uno de los biomas más amenazados del mundo (Redford et al. 1990; Janzen 2002). El Gran Chaco Americano es una región que abarca más de 1.000 .000 de $\mathrm{km}^{2}$ en el centro de América del Sur y se extiende en territorios de cuatro países: Argentina (62,19\%), Paraguay $(25,43 \%)$, Bolivia $(11,61 \%)$ y Brasil $(0,77 \%)$. Posee un amplio gradiente climático y características geológicas únicas que generan una gran diversidad de ambientes: extensas Ilanuras, sabanas secas e inundables, esteros, bañados, salitrales, y una gran extensión y diversidad de bosques primarios y secundarios. Todo esto se traduce en una alta diversidad de especies que hacen del Chaco un área clave para la conservación (Morello y SaraviaToledo 1959). Sin embargo, grandes extensiones de bosque chaqueño han sido destruidos o degradados como consecuencia a las presiones económicas, especialmente en Argentina y Paraguay (Morello y Saravia-Toledo 1959; Morello y Hortt 1985; Schofield y Bucher 1986; Hansen et al. 2013).

Afortunadamente y gracias a la alianza entre actores locales (Capitanía del Alto y Bajo Isoso, CABI) y conservacionistas (Wildlife Conservation Society, WCS) se logró la creación del Parque Nacional Kaa-lya cuya área es de $34.000 \mathrm{~km}^{2}$. Con el decreto promulgado se protegió el 22\% del Chaco boliviano, y una de las representaciones de bosque tropical seco más grandes del mundo. Además, contiene una alta diversidad biológica incluyendo especies endémicas. A su vez, este área protegida es fuente de recursos para tres grupos étnicos autóctonos: Guarani-Isoceños, Chiquitanos y Ayoreodes; es parte de las jurisdicciones de cuatro municipios; y colinda con la frontera norte del Paraguay (Taber et al. 1997). Con una población humana casi inexistente que incluye apenas un grupo de Ayoreodes en aislamiento voluntario y unos pocos puestos ganaderos y militares, virtualmente sin caminos de acceso, el Kaa-lya es una de las últimas grandes áreas silvestres del mundo (Noss et al. 2002).

La declaración original del parque incluye un área de protección estricta y tres áreas de manejo integrado, este / norte / oeste, anticipados como áreas de aprovechamiento de parte de los pueblos indígenas presentes en la zona. Sin embargo, en base a las extensas investigaciones ecológicas y socio-económicas en el campo y la amplia participación, el Plan de Manejo del Parque Nacional Kaa-lya describe y demarca una zonificación actual que permite varios niveles de uso de recursos naturales en diferentes porciones del área protegida (Navarro y Fuentes 1999; Castillo et al. 2006); siguiendo las zonificaciones previas del Proyecto Kaa-lya/SERNAP (2000, 2001): 1) Zonas de amortiguamiento externas: para absorber impactos en secciones frágiles del área protegida. 2) Zonas de uso intensivo extractivo: actividades productivas consistentes con el desarrollo sostenible, sujetos a un control y reglamentación estricto; investigación científica (ecología del fuego, forrajes naturales, hidrología, limnología, acuíferos, evaluación de recursos de fauna); ganadería extensiva sostenible, mejoramiento de forrajes naturales; cacería, pesca y recolecta de subsistencia; monitoreo; uso público con ecoturismo y educación ambiental. 3) Zonas de uso extensivo extractivo: aprovechamiento y manejo de recursos naturales, a través de la extracción y recolecta regulada de productos naturales (madera de construcción, postes, leña, cacería y pesca de subsistencia por parte de pueblos indígenas); investigación científica aplicada al manejo y monitoreo de recursos naturales; ecoturismo de bajo volumen y educación ambiental. 4) Zonas de 
uso extensivo no-extractivo: protección del ambiente natural con un mínimo de impacto humano, con actividades limitadas a la investigación científica, monitoreo y ecoturismo controlado.

Contexto institucional. Durante los años 1980, cuando los gobiernos de Bolivia y Alemania acordaron diseñar e implementar un Plan de Protección de Recursos Naturales para el departamento de Santa Cruz, la organización líder $\mathrm{CABI}$, representando al pueblo indígena mayoritario, demandó la tenencia de territorio en el Gran Chaco, bajo la propuesta de creación de un área protegida respondiendo a la visión de que este área aseguraría la sobrevivencia de los pueblos originarios del Chaco. La propuesta fue basada en la idea de promover alternativas a las actividades productivas que dominaban la economía regional como la agricultura y la ganadería extensiva (Arellano 2003).

La idea de las actividades alternativas estuvo basada en el desarrollo económico, el mismo que preveía dos preocupaciones principales (Winer 2003): 1) que el crecimiento económico sea equitativo; 2) que el crecimiento económico no acarreara un alto costo medioambiental, definido como deforestación, degradación de suelos, destrucción de hábitats de especies clave. Finalmente en 1997 la demanda de territorio fue aprobada bajo la propuesta de la creación del Parque Nacional Kaa-lya. El parque fue declarado el primer área protegida en las Américas co-gestionado entre el gobierno central y un pueblo indígena (Taber et al. 1997). La coadministración del área protegida por la CABI tuvo un periodo de duración de 10 años. Además, en el contexto de gobernanza e institucionalidad, la participación activa de los pueblos en la administración de las áreas protegidas en Bolivia se lleva a cabo mediante la activación del Comité de Gestión del área protegida. El comité de gestión es el órgano representativo de la población local que participa en la planificación y coadyuva en la fiscalización de la gestión del área protegida (el Parque Kaa-lya en este caso).

El reto luego de la creación del área protegida fue dar trascendencia a un logro puramente político, para darle un manejo científico y administrativo. Este objetivo culminó en la elaboración del "Proyecto Kaa-lya", conjuntamente implementado desde 1997 hasta 2003 por CABI y WCS con el apoyo monetario de la United States Agency for International Development (USAID/Bolivia). Uno de los objetivos fundamentales de la $\mathrm{CABI}$, en el contexto del Proyecto Kaa-lya, fue promover la formación técnica de pobladores locales en las diferentes áreas (recursos naturales, educación ambiental, fortalecimiento institucional) de trabajo de dicho proyecto. Esto se tradujo en la idea de contar con "parabiólogos" (dentro del área de recursos naturales) modificando la idea de Daniel Janzen en Costa Rica con los "para-taxónomos" (Janzen 2004). Los parabiólogos en este caso son indígenas de la región del Chaco, elegidos por sus comunidades.

Son conocedores de la realidad local (biológica y social) de sus zonas de vida. Siendo usuarios de los recursos naturales, mayormente cazadores y pescadores, los parabiólogos son personas óptimas para recibir entrenamiento en técnicas específicas en la colecta de datos científicos dado el conocimiento nato sobre historia natural y su rol dentro de la sociedad que representan. Dentro de este enfoque de conservación, investigación y participación local, la alianza CABI/WCS ha trabajado activamente en el Chaco cruceño por más de una década, y durante este tiempo logró: 
Formar a más de 20 técnicos locales en investigación/manejo de recursos naturales y en educación ambiental. Este entrenamiento desencadenó en que más de 700 cazadores, 2.700 niños de escuelas y sus maestros, además 5.000 mujeres quedaron involucradas en el programa a través de la asociación de mujeres guaraníes.

Asistir en la preparación e implementación del plan de manejo para el Parque Nacional y Área Natural de Manejo Kaa-lya del Gran Chaco, proveyendo apoyo técnico y científico.

Guiar en el proceso de fortalecimiento institucional de su CABI, con el fin de fortalecer el brazo técnico administrativo, capaz de asumir los retos del co-manejo del Parque Kaa-lya y la Tierra Comunitaria de Origen (TCO) Isoso, además de involucrarse en la propuesta de zonificación y manejo para la TCO.

Contexto biológico (enfocado en mamíferos). La esencia del trabajo de WCS en el paisaje del Gran Chaco se enfocó principalmente en la conservación de especies endémicas y en peligro de los bosques secos, así como también en el estudio de especies de caza (Noss et al. 2004a) importantes en la dieta de los grupos étnicos de la zona. El Gran Chaco cuenta con una gran diversidad de especies, entre ellas al menos 10 especies de mamíferos endémicos. El Gran Chaco también fue escenario de la cacería comercial de gatos silvestres, zorros, pecaríes, entre otros, durante las décadas 1970 y 1980, lo cual indica una explotación histórica no muy bien documentada de cacería. Esto último tuvo impactos severos sobre las poblaciones de mamíferos en el Chaco boliviano (Taber 1991).

La estructura de trabajo dentro del contexto del estudio de los mamíferos incluyó esfuerzos para documentar la situación del guanaco chaqueño (Cuéllar et al. 2005); investigación sobre la ecología del jaguar y sus presas (Maffei et al. 2004); la conservación y uso de especies paisaje (Noss y Cuellar 2008) (incluyendo el endémico pecarí chaqueño); la resolución de conflictos entre humanos y vida silvestre; esfuerzos para entender los cambios estructurales en el paisaje realizando estudios en ecología del fuego (Navarro 2002); y finalmente el desarrollo de esfuerzos binacionales (Bolivia-Paraguay) para el relevamiento de mamíferos (Romero-Muñoz et al. 2010) y el entrenamiento de parabiólogos (Hesse y Cuéllar 2008).

Tomando en cuenta los tres contextos arriba mencionados, en este trabajo resumiremos lo referente a las investigaciones sobre mamíferos logradas en un marco de inclusión y colaboración directa con los actores locales y parabiólogos. Finalmente haremos un recuento de los logros obtenidos por la alianza CABI/WCS y las perspectivas de continuación de los esfuerzos de conservación netamente locales.

\section{Desarrollo del programa de estudio y conservación de los mamíferos del Chaco}

Estudios sobre especies amenazadas. Entre los mamíferos, las especies amenazadas según criterios internacionales de la Unión Internacional para la Conservación de la Naturaleza (UICN 2010) y nacionales de Bolivia (Aguirre et al. 2009) incluyen el guanaco chaqueño Lama guanicoe, el jaguar Panthera onca, el solitario o pecari chaqueño Catagonus wagneri, y el pejichi o armadillo gigante Priodontes maximus. A 
nivel local, se acordó una veda indefinida desde 2005 para dos especies en peligro de extinción, el guanaco y el solitario (Barrientos y Cuéllar 2009).

a. Guanaco, Lama guanicoe

El guanaco es el ungulado más grande y la especie de mamífero más amenazada en el paisaje Kaa-lya. El conocimiento sobre esta especie es limitado, sin embargo la información que hemos podido acumular a lo largo de una década sobre la biología y ecología de la especie es actualmente lo que se conoce a nivel nacional.

El guanaco fue denominado "En peligro", debido a la reducción de sus poblaciones y su área de distribución; luego "Extinto" refiriéndose a las poblaciones de las tierras altas de Bolivia; y actualmente "En peligro critico" debido a que las poblaciones presentes son relictuales y aisladas, además de estar amenazadas por la cacería y pérdida del hábitat (Cuéllar y Núñez 2009).

La distribución conocida del guanaco en el Chaco boliviano es de 61,250 hectáreas (uniendo los puntos extremos de observaciones). Estas observaciones fueron hechas durante cuatro censos aéreos entre 1999 y 2011, y luego confirmadas por 484 observaciones directas en muestreos desde tierra (Cuéllar 2011). Además de las observaciones directas obtuvimos indicios de presencia de la especie mediante el monitoreo sistemático de revolcaderos, letrinas, y huellas. Adicionalmente, obtuvimos registros fotográficos mediante trampas cámara utilizadas para el monitoreo de jaguares (Cuéllar 2011). El primer muestreo sistemático con trampas cámara confirmó la presencia de guanacos dentro del Parque Nacional Kaa-lya, registrando dos individuos nuevos que las observaciones directas en el campo no habían identificado previamente.

En cuanto a la estructura de la población identificamos tres formas de organización social: grupos de adultos solamente, grupos familiares (con crías), e individuos solitarios (tanto hembras como machos). El tamaño de grupo promedio fue de 2.8 individuos. Los solitarios (siendo estos adultos o juveniles, así como hembras y machos) fueron encontrados más frecuentemente que grupos con más de dos individuos. Las pariciones y la expulsión de juveniles de los grupos familiares fueron concentrados en la época Iluviosa (Cuéllar 2011).

Nuestras observaciones se concentraron entre las 6:00 y las 18:00 hrs. De un total de 210 observaciones, 166 fueron de animales comiendo o caminando (94\% de las observaciones de animales comiendo fueron registradas en lugares abiertos con vegetación baja, principalmente con presencia de gramíneas) mientras que 44 fueron de animales descansando alrededor del medio día (en 77\% de las observaciones registramos animales protegiéndose del sol bajo la sombra de uno o varios árboles).

Los parabiólogos adoptaron el método de reconocimiento por marcas y características externas de los animales de cada grupo, dado que no pudimos utilizar ningún método invasivo para monitorear a la población. Utilizando el método de reconocimiento de individuos estimamos el área promedio de acción de seis grupos monitoreados que fue de $24 \mathrm{~km}^{2}$ ( $\pm 14 \mathrm{SD}$ ) con un rango de entre $13-51 \mathrm{~km}^{2}$.

Realizamos un estudio de dieta comparativo (guanaco-vaca) siguiendo el procedimiento de preparación de muestras fecales (Williams 1962) para luego ser sometidas a un análisis microhistológico de identificación (Baumgartner y Martin 1939). Encontramos que el guanaco consumió 53 de las 57 especies de plantas consumidas 
por la vaca, aunque solo algunas especies fueron encontradas con mayor frecuencia que otras. Mediante un análisis de similaridad (ANOSIM) basado en la frecuencia de especies notamos una separación muy clara a nivel de las especies consumidas $(r=0.52$, $P=<0.0001$ ), así como también a nivel de grupos ecológicos (gramíneas, suculentas, herbáceas, trepadoras, y arbóreas) $(r=0.3312, P=<0.001)$. Las especies consumidas por guanacos (29 muestras) fueron principalmente: Urvillea chacoensis (trepadora; 100\%), Ximenia americana (arbórea; 86\%) y Celtis chichape (arbórea), Angelphyton pseudosilphioides (herbácea), y Aristida mendocina (gramínea; 83\%) cuya distribución de esta última se ha reducido en un 95\% en los últimos 40 años (Pinto 2005). Por otro lado, las especies consumidas por vacas (25 muestras) fueron principalmente: Chloris castilloniana (gramínea; 100\%), seguida por la especie arbórea colonizadora de las áreas abiertas Pithecellobium chacoense (96\%), y luego Agonandra sp., A. pseudosilphioides, y $U$. chacoensis (las tres especies $80 \%$ ).

Basados en un periodo de 10 años sugerimos que ha habido una retracción en la distribución del guanaco y esto ha venido ocurriendo en los últimos años debido probablemente a la perdida de hábitat, traducido a perdida de zonas abiertas dominadas principalmente por la gramínea A. mendocina. Adicionalmente, la cacería furtiva ha sido otro de los factores que podrían haber causado la reducción de la población. Sin embargo, en el año 2001 y gracias a la presencia casi permanente de parabiólogos en la zona, y de su comunicación constante con los dueños y encargados de estancias, logramos proteger la población de guanacos de la presión por parte de cazadores furtivos.

La única población de guanacos en Bolivia se encuentra vinculada con la recientemente confirmada población Paraguaya de guanacos (Villalba 2004). Ambas poblaciones son pequeñas y fragmentadas, y por lo tanto altamente vulnerables. Es por esto último que desde el año 2001 y hasta el presente intentamos fortalecer los vínculos con las instituciones y profesionales socios en Paraguay. Además, empezamos a trabajar con ganaderos para asegurar que sus alambrados no aislaran a los grupos entre sí, o que no impidieran los desplazamientos estacionales o dispersión de juveniles, manteniendo corredores viables. Sin embargo, el mantenimiento de alambrados es vital para contener al ganado en potreros y evitar que el ganado utilice zonas dentro del parque. Los parabiólogos Isoseño-Guaraní fueron clave para el éxito en el Paraguay: ellos lideraron los trabajos de campo en Bolivia, y dado que hablan el mismo idioma que en las comunidades indígenas paraguayas tuvieron una excelente aceptación en la porción paraguaya de la distribución del guanaco. Los parabiólogos han identificado las amenazas principales y desarrollado respuestas prioritarias a esas amenazas. Fueron parte fundamental en el programa de conservación del guanaco en la porción boliviana, habiendo también compartido sus esfuerzos y éxitos en congresos regionales e internacionales de manejo de fauna (Segundo 2007). Además, los dirigentes Isoseños y la comunidad Isoseña más cercana al área de distribución del guanaco (Isiporenda) han manifestado reiteradas veces su intención de crear una reserva comunitaria para proteger a los guanacos, una vez completada la titulación de las tierras comunales y privadas colindando el área protegida en el 2005. Esto último aún no se ha concretado, sin embargo siguen las negociaciones cada vez que cambian las autoridades en el municipio al cual pertenecen las comunidades de Isoso (Cuéllar et al. 2005). 


\section{b. Jaguar Panthera onca}

El jaguar es una especie carismática que figura en muchos cuentos isoseño-guaraníes, y es una especie bandera para el Parque Nacional Kaa-lya. Al mismo tiempo es perseguido por los ganaderos de la región quienes se encuentran en todo el Chaco fuera del parque, aunque también hay algunos puestos ganaderos al interior del área protegida. Por daños reales o imaginados, en cuatro años se cazaron 347 jaguares en 85 puestos ganaderos del Chaco, Chiquitanía, y Pantanal del Departamento de Santa Cruz (Arispe et al. 2009). Sin embargo, existe un interés compartido entre estos actores para definir cómo asegurar la ganadería y al mismo tiempo la conservación del jaguar en el paisaje.

La principal metodología que utilizamos para el estudio de jaguares en el Parque Nacional Kaa-lya ha sido el muestreo sistemático mediante trampas-cámara, según el protocolo detallado preparado por WCS (Silver 2004). Los individuos de jaguar fotografiados en las trampas-cámara se identifican por sus patrones únicos de manchas.

La densidad poblacional se estima aprovechando de manera explícita la información de las capturas y recapturas en combinación con la ubicación espacial de las capturas (Borchers y Efford 2008). Realizamos en total 13 muestreos sistemáticos con trampascámaras en seis zonas de investigación: Tucavaca, Palmar de las Islas, Ravelo, Cerro, Guanaco y Estación Isoso, dentro del paisaje Kaa-lya entre 2001-2007. Uno de los muestreos fue la combinación entre dos sitios Palmar de las Islas y Ravelo. Los muestreos de Palmar de las Islas y Palmar-Ravelo agregaron 2-3 estaciones en una propiedad ganadera privada en Paraguay. Tucavaca, Ravelo, y Palmar de las Islas se ubican en bosques transicionales Chaco-Chiquitanos, con precipitaciones anuales decreciendo de 800 mm en Tucavaca, a 650 mm en Palmar de las Islas-Ravelo. Estación Isoso se ubica en el bosque transicional Chaco-Amazonía (650 mm de precipitación anual). Cerro se encuentra en un bosque chaqueño seco (500 mm), mientras que Guanacos presenta una combinación de bosque seco chaqueño y pampas chaqueñas (400 mm). Los muestreos abarcaron polígonos de cámaras de 48 a $434 \mathrm{~km}^{2}$, con estaciones de cámaras (un promedio de 30 pares) distantes entre sí por 1 a $4 \mathrm{~km}$, y duraron entre dos y tres meses.

El trabajo estuvo supervisado por biólogos y parabiólogos, quienes implementaron los muestreos, la instalación y revisión de cámaras, análisis de datos y la presentación de resultados en congresos. A pesar de las limitaciones logísticas y de acceso, los muestreos nos permitieron estimar una densidad poblacional entre $0,4-2,5$ individuos $/ 100 \mathrm{~km}^{2}$ en todos los sitios.

En el parque se estima una población de jaguares en unos cientos de individuos. Los muestreos en Palmar de las Islas nos permitieron confirmar además desplazamientos de jaguares entre Paraguay y Bolivia, aparentemente con áreas de acción que abarcan porciones de los dos países. Es un hecho sin duda común, pero pocas veces documentada (Romero-Muñoz et al. 2007).

Por otro lado, la combinación de trampas-cámara y observaciones directas de jaguares en Estación Isoso nos permitieron confirmar la presencia de una hembra (Kaaiyana y sus crías) a lo largo de más de seis años, con crías tanto en 2006 como en 2011 (Cuéllar et al. 2012). El acceso al derecho de vía del gasoducto, el mismo que abarca unos $130 \mathrm{~km}$ al norte del PN Kaa-lya, está controlado por personal del gasoducto y por guardaparques para evitar cualquier intento de cacería o colonización. El tráfico vehicular se limita 
estrictamente en cuanto número y tipo de vehículo y número de pasajeros; y se limita a horas diurnas y condiciones secas. Los registros consecutivos de jaguares hacen suponer que estos se han adaptado a la presencia de la estación de compresión y las actividades humanas asociadas con ella. El trabajo de monitoreo coordinado entre los guardaparques en el PN Kaa-lya, en colaboración con WCS y la Fundación Kaa-lya, permitirá a futuro la promoción del ecoturismo.

Finalmente, según las entrevistas realizadas a representantes de 28 propiedades en el Chaco, estas abarcan una extensión entre 300 - 20.000 ha con hatos de entre 80 1000 cabezas de ganado (Arispe et al. 2009). La ganadería en la zona es extensiva, con animales sueltos aprovechando la vegetación natural y con la provisión de agua solo en los sitios y las épocas más secas. Las prácticas de manejo como ser la rotación de potreros, control de la reproducción, y medidas veterinarias más allá de la vacunación son pocas o inexistentes. Se mantienen pocos registros sobre mortalidad y productividad. La mitad de los ganaderos reporta pérdidas por jaguares, aunque las consideran aceptables dadas su prácticas de manejo la mayoría a su vez indica que el método preferido para responder a este tema es matar el jaguar o ahuyentarlo (Arispe et al. 2009). WCS promovió un taller en 2006 con ganaderos del Departamento para discutir y promover alternativas de manejo para reducir conflictos entre jaguares y ganado, y produjo materiales para que los puesteros identificaran las causas de la mortalidad del ganado (Cuéllar y Combès 2002; Arispe et al. 2005).

\section{c. Solitario Catagonus wagneri}

Promediando unos $35 \mathrm{~kg}$ y $57 \mathrm{~cm}$ de alto a la cruz (Eisenberg y Redford 1999), este es el más grande de los pecaríes y se creía extinto hasta su re-descubrimiento en Paraguay en el 1972 (Wetzel et al. 1975). Subsecuentemente se confirmó su endemismo en las regiones chaqueñas secas de Bolivia y Argentina. Sin embargo, la cacería, la conversión de hábitat, y posiblemente las enfermedades han reducido su área de distribución (Taber 1991). La población global se estima en menos de 5000 individuos, y la especie se considera amenazada según la UICN (Altrichter et al. 2008) y la Lista Roja de Bolivia (Aliaga-Rossel et al. 2009). De hecho, la presencia de esta especie amenazada y endémica en el Chaco boliviano fue uno de los justificativos para la creación del enorme Parque Nacional Kaa-lya (Taber et al. 1997). Forman manadas muy pequeñas (por eso el nombre local en Bolivia de "solitario") en comparación con las otras dos especies de pecaríes, promediando 3 individuos por grupo (Cuéllar y Noss 2003).

Hasta el 2005, los únicos registros dentro del paisaje Kaa-lya fueron de cazadores, reportando 22 animales cazados entre 1997 y 2007 en el Isoso. Sin embargo, con las fotos obtenidas con trampas-cámara desde el 2005 ampliamos los lugares con registros de esta especie: en el campamento de guardaparques del PN Kaa-lya en Charata; en los Bañados de Isoso (bosque chaqueño estacionalmente inundado); en la Estación Isoso (bosque transicional Chaco-Chiquitano); en Palmar de las Islas (sabanas con palmeras estacionalmente inundadas); y Guanacos (pampas o pastizales sobre suelos sueltos arenosos). Gracias a la participación activa de los guardaparques y parabiologos, con base en el campamento Charata, se obtuvo información valiosa sobre esta especie en este sitio (Banegas y Maffei 2007). Por otro lado, como producto de la información generada por cazadores y parabiólogos sobre la especie, las comunidades adoptaron 
una medida de conservación prohibiendo la cacería de esta especie (Noss y Cuéllar 2001; Banegas y Maffei 2007).

\section{d. Pejichi Priodontes maximus}

Aparentemente limitados por niveles de precipitación, la distribución de $P$. maximus ocupa las porciones boreales y orientales del Parque Kaa-lya (Cuéllar y Noss 2003; Tarifa 2009; Noss et al. 2010a). A pesar de su tamaño, 30 kg o más, y aunque deja cuevas enormes abandonadas como evidencia de su presencia, sigue siendo una especie difícil de observar y por ello se considera una especie rara. Esto se debe también a la combinación de sus hábitos nocturnos y fosoriales.

Luego de haber observado indicios de la presencia de esta especie (huellas y cuevas activas) recién en el 2001 obtuvimos los primeros registros fotográficos mediante la utilización de trampas-cámara. Con un esfuerzo de más de 27.500 trampas-noches en cinco sitios, obtuvimos 34 registros de 24 individuos de pejichi dentro del paisaje Kaa-lya, pero solo en Tucavaca, Estación Isoso, y Ravelo. Como lo habían indicado los cazadores y la ausencia de indicios, no registramos ningún registro fotográfico en Cerro Cortado o en Guanacos. Los patrones únicos de escamas permiten la identificación de individuos, así como la línea divisora entre escamas oscuras y claras en el caparazón y en la pata trasera fue especialmente útil, tal como fué el número de escamas por fila desde el borde inferior del caparazón hasta la línea divisora. En base a esas diferencias, identificamos 18 individuos en Tucavaca (8 machos, 1 hembra, y 9 sin sexo identificado). Según los modelos de captura-recaptura espacialmente explícitos, la densidad poblacional de pejichis en Tucavaca podría alcanzar los 5 individuos/100 km² (Noss et al. 2004b).

Estudios sobre especies importantes para el consumo local. Estas especies son prioridad más del lado comunitario—de las comunidades isoseñas y de la CABI—por sus aportes de subsistencia y comercial. El interés comunitario compartido con los conservacionistas es el de asegurar el uso sostenible a largo plazo de este recurso. Entre los mamíferos, las especies más importantes incluyen los ungulados (la urina -Mazama gouazoubira, el taitetú o pecarí de collar Pecari tajacu, el tropero o pecarí de labios blancos Tayassu pecari, y el anta o tapir Tapirus terrestris) y los armadillos (el tatú mula o tatú de nueve bandas Dasypus novemcinctus, el peji o armadillo de seis bandas Euphractus sexcinctus, el tatú pecho amarillo Chaetophractus villosus, el tatú llorón C. vellerosus, y el corechi o tatú bola Tolypeutes matacus) (Cuéllar 2000b).

El programa de investigación y manejo comunitario de fauna en la zona de Isoso se inició en el 1996 con la participación de cazadores y un equipo de parabiólogos Isoseños.

Con el fin de generar información sobre las poblaciones de fauna en la zona y sobre la cacería de subsistencia y comercial, se aplicaron las siguientes metodologías: el automonitoreo de cacería, análisis de datos de cacería, el monitoreo de fauna por transectas, el monitoreo de fauna mediante registro de huellas en parcelas, y conteos mediante batidas y muestreos sistemáticos con trampas-cámara. Estos datos se analizaron a través de modelos para evaluar la sostenibilidad de la cacería de subsistencia, y se discutió en las comunidades los resultados técnicos en relación a sus creencias y conocimientos tradicionales para generar y aplicar propuestas y medidas de manejo con el fin de 
asegurar el uso sostenible de la fauna. Los parabiólogos lideraron varios aspectos del programa: el trabajo con los cazadores de sus respectivas comunidades, la toma de datos en transectas, el análisis de datos, actividades de educación ambiental, y las discusiones tomadas con las comunidades (Noss et al. 2005).

En el auto-monitoreo de cacería participaron en total más de 700 cazadores (entre 100 y 350 cada año) y 33 puestos ganaderos aportando datos sobre especies cazadas (sexo, peso, estado reproductivo, y edad aproximada), lugares de cacería, esfuerzo (horas o días, con qué arma, con o sin perros). En total se cazan 29 especies de mamíferos, pero las más importantes en biomasa y número de presas son las cuatro especies de ungulados y las cinco especies de armadillos mencionados arriba. Solo entre 20022007 los registros suman más de 7.000 ungulados y armadillos (Noss 1998, 1999; Cuéllar 2000a; Noss 2000; Noss et al. 2003a).

Adicionalmente, registramos de forma mensual la actividad de los cazadores "potenciales" (jóvenes y hombres adultos) para definir la proporción de cazadores activos participando en el programa de auto-monitoreo, y así extrapolar la cosecha total en Isoso según los datos reportados por los cazadores voluntarios. A través de los mismos datos se estimó un índice de "capturas por unidad de esfuerzo" (catch per unit effort - CPUE), dividiendo el total de capturas por especie entre el número de cazadoresmeses. Estimamos que 2/3 de los cazadores activos participaron en el sistema de automonitoreo (Noss et al. 2003a; Cuéllar et al. 2004; Noss et al. 2004a; Noss et al. 2005; Noss et al. 2008). Además los cazadores aportaron cráneos, muestras estomacales y tractos reproductivos para describir la estructura poblacional, dieta, y reproducción de algunos mamíferos respectivamente. En el caso de los ungulados, especímenes de cráneos permitieron determinar la edad de los animales cazados en base al desgaste dental (Maffei 2000, 2001, 2003, 2004; Rojas-Suárez y Maffei 2004), generar claves de desgaste dental para urina, taitetú, tropero y anta; establecer la relación entre el desgaste observado y la edad según los anillos dentales; y analizar cráneos provenientes de la cacería en Isoso para detectar si hubo cambios en la distribución de edades.

La urina y el taitetú fueron los ungulados más cazados, pero el número de animales cazados se mantiene en el tiempo y se los encuentra cerca de las comunidades. En general los datos de abundancia (según batidas, trampas cámara, y huellas) coinciden, sugiriendo un uso sostenible de las mismas. Las antas y los troperos, por su lado, son presas menos comunes, y no se encuentran durante todo el año, además son menos abundantes en áreas con cacería (Cuéllar y Noss 1997; Noss y Cuéllar 2000; Noss et al. 2003a; Noss et al. 2006). Los modelos estándares para evaluar la sostenibilidad de la cacería, comparando el estado de las poblaciones de fauna (su abundancia y productividad en su hábitat natural) con el uso de las mismas especies por parte de pobladores locales, también indican que la cacería de urinas y de taitetús en Isoso es sostenible. Los mismos modelos y las observaciones de los cazadores Isoseños sugieren una sobre-explotación de anta y de tropero en Isoso (Noss 2000; Noss y Cuellar 2008).

El uso actual para fines de subsistencia aparentemente es sostenible para las cinco especies de armadillos, con la excepción de T. matacus y, posiblemente, C. vellerosus. Dentro de las cinco especies de armadillos reportadas, T. matacus es la especie cazada con mayor frecuencia. Esto último sumado a que tiene la productividad más baja de todos los armadillos (una cría por gestación), y una estrategia de defensa que puede ser 
efectiva contra jaguares y zorros (Pseudalopex gymnocercus y Cerdocyon thous) pero no contra cazadores humanos, aumenta su vulnerabilidad en zonas con presión de cacería.

Los armadillos diurnos, el peji y el pecho amarillo, son menos vulnerables a cazadores Isoseños porque los cazadores y sus perros evitan las horas más calientes del día. El tatú mula tiene una productividad relativamente alta (cuatro crías idénticas por gestación) y resiste mejor la presión de cacería (Noss 2000; Noss y Cuellar 2008).

Como parte de las estrategias de conservación adoptadas por los actores locales, las comunidades y autoridades de Isoso han desarrollado y adoptado un reglamento de cacería que incluye vedas temporales (para tropero y anta) entre otras medidas; una zonificación interna creando reservas comunales sin cacería; y planes de manejo para el uso comercial sostenible de taitetú (Noss et al. 2010b). En esta región, el único mamífero sujeto a la cacería comercial hoy en día es el taitetú, por la venta de su cuero.

Los estudios anteriores de cacería y de abundancia sirvieron de base técnica para una propuesta de manejo comercial de la especie. El "Plan de manejo para el comercio de cueros de taitetú (Tayassu tajacu) producto de la cacería de subsistencia de las comunidades del Isoso"(Cuéllar y Noss 2002) fue aprobado por parte de la Dirección General de la Biodiversidad a partir de 2006 (con extensiones válidas hasta 2011) e implementado por la Asociación de Cazadores y Comercializadores de Cueros de Peni y Taitetú en el Isoso. Este plan de manejo implica un compromiso serio por parte de los pobladores Isoseños para dar cumplimiento a las normas establecidas en el plan: estudios de mercado, muestreo sistemático y constante de la población de cada especie, establecimiento y control de cuotas anuales por especie, conformación de un comité de gestión, y capacitación de técnicos y cazadores (Noss et al. 2003b; Rumiz 2004).

Estudios sobre recuperación de hábitats. Dado que más de la tercera parte de los tres millones de hectáreas que conforman el parque Kaa-lya han sido afectadas más o menos recientemente por el fuego, este tema fue motivo de estudios de cara a la elaboración del plan de manejo del área protegida en el 2001. Conformamos un equipo multidisciplinario con el objetivo de crear una línea base de información que nos permitiese entender la dinámica de los ecosistemas influenciados por el fuego con relación a la diversidad actual (Navarro 2002). Este estudio de ecología del fuego evaluó la diversidad biológica en zonas afectadas, así como la capacidad de producción ganadera en estas áreas, tomando en cuenta el fuego como potencial herramienta de manejo. Con relación a mamíferos, el rol del fuego estuvo relacionado directamente al área de distribución del guanaco. Según las encuestas realizadas los cazadores indígenas usaban históricamente el fuego en el Chaco para mantener formaciones de sabanas y facilitar la cacería. Los ganaderos utilizan el fuego para promover el crecimiento y rebrote de pastos para el ganado. Sin embargo, tanto comunidades indígenas como ganaderos están perdiendo su conocimiento tradicional del uso del fuego para manejar ecosistemas y aumentar su biodiversidad y productividad. El estudio de la ecología del fuego busca recuperar prácticas tradicionales, optimizar la producción de ganado, entender las respuestas de la fauna y de los ecosistemas chaqueños al fuego, en particular las relaciones entre el fuego y la conservación de guanacos, para así generar propuestas de conservación y monitoreo a largo plazo. 
El fuego incrementa la heterogeneidad del hábitat, pero disminuye la diversidad del hábitat en una escala de paisaje (Navarro 2002). El fuego también reduce la diversidad estructural en todos los tipos de vegetación con la excepción de las sabanas de Isiporenda. En estas parece que el uso continuo y sistemático del fuego, en combinación con la ausencia actual de ganado, han generado una adaptación de la estructura vegetal al fuego con una abundancia excepcionalmente alta de plantas resistentes al fuego como son Acacia aroma, Arachis batizocoi, Caesalpinia stuckertii, Rhynchosia burkartii, y Senna chloroclada (Fabaceae), Abutilon herzogianum (Malvaceae), Annona nutans (Annonaceae), Gymnocalycium marsoneri (Cactaceae), y Schinopsis cornuta (Anacardiaceae).

Entre los mamíferos grandes, algunas especies son más abundantes en áreas alteradas por el fuego tanto en el sur-oeste como en el este del parque: el taitetú, la urina, el zorro patas amarillas Pseudalopex gymnocercus, el peji, el corechi, y el conejo Sylvilagus brasiliensis. El guanaco es la especie bandera de las sabanas al sur-oeste del parque Kaa-lya. Las especies de mamíferos raros o ausentes en las áreas abiertas o con mayor incidencia de fuego son el ocelote Leopardus pardalis, el anta, y los primates. Entre los mamíferos pequeños, los marsupiales son más abundantes en hábitats de bosque, mientras que los roedores son más abundantes en hábitats alterados por el fuego. Por ejemplo el ratón del Chaco de Pearson Andalgalomys pearsoni es endémico de las pampas chaqueñas (Olds et al. 1987).

En general, los incendios se originan principalmente en los puestos ganaderos, afectan áreas pequeñas, y se desplazan gracias a vientos del norte o del sur. En el suroeste del PNKI los incendios datan del siglo 18 y la introducción del ganado a la zona por los españoles, pero en el este los incendios son mucho más recientes, post1970. Es entonces difícil separar los efectos del fuego de los del ganado. El fuego no-manejado, intermitente y ocasional genera estructuras de vegetación con menos capacidad forrajera (biomasa viva y muerta) para el ganado y la fauna. Esta condición se agudiza con la sobre-carga por falta de manejo del ganado. Por otro lado, un régimen de fuego manejado con cargas ganaderas consistentes con la capacidad de carga del área genera estructuras productivas de vegetación de pampas como las encontradas en Isiporenda con una diversidad sorprendentemente alta de paisajes, estructuras de vegetación, y fauna (Navarro 2002).

Capacitación de parabiólogos en el estudio de mamíferos. El proyecto Kaa-lya tuvo un fuerte componente de capacitación de recursos humanos. El objetivo específico de capacitación local en el área de investigación aplicada se enfocó en la formación de parabiólogos como parte del establecimiento de un programa de investigación, el cual debería garantizar sitios estratégicos de estudio y la colecta continua de datos sobre población y ecología de especies silvestres clave. Estos estudios específicos deberían de proveer una línea de investigación enfocada a contribuir en la implementación de los planes comunitarios de manejo y conservación en la región. Para este propósito el programa desarrolló diferentes áreas de trabajo: 1) investigación aplicada, 2) investigación participativa sobre población y ecología de animales silvestres, y la definición de prácticas de manejo de vida silvestre; y 3) obtención e interpretación de datos para el manejo de recursos naturales con la colaboración activa de el componente "educación 
ambiental" del proyecto. Este último punto tuvo el objetivo de difundir las actividades de investigación/conservación llevadas a cabo por los parabiólogos y con esto crear vínculos con actores y organizaciones gubernamentales locales.

Los parabiólogos también participaron activamente del esfuerzo transfronterizo de conservación entre Paraguay y Bolivia a través de CABI/WCS y Fundación DesdelChaco.

Este acuerdo resulto en: a) la producción de un mapa de vegetación para la región abarcando estos dos países (Navarro 2002); b) un acuerdo para complementar estudios socioeconómicos comparativos; c) estudios complementarios y comparativos para el guanaco; y d) la creación de un área protegida contigua al Parque Kaa-lya en Paraguay.

Una prueba de éxito del periodo de capacitación de parabiólogos fue el hecho de la participación activa en congresos internacionales presentando los resultados de sus propias investigaciones. Esto aseguró el sentimiento de apropiación en el proceso de investigación y conservación realizado.

Como parte de sus responsabilidades de investigadores, los parabiólogos y monitores tuvieron un papel importante en la difusión de las actividades desarrolladas en las comunidades, principalmente como facilitadores en talleres comunitarios. En el 2002, como iniciativa propia, los mismos parabiólogos establecieron un programa de visitas por estudiantes y docentes del Isoso al campamento Cerro Colorado, donde los parabiólogos presentaban su trabajo directamente a miembros de las comunidades.

En resumen se cumplieron los objetivos planteados por el proyecto Kaa-lya en términos de investigación; producción y difusión de información biológica/ecológica (Cuéllar y Noss 2003); entrenamiento de gente local en la colección de datos y su interpretación; así como también el fortalecimiento de vínculos con las comunidades y las organizaciones gubernamentales. Sin embargo, cuando los fondos destinados a este proyecto llegaron a su fin, los parabiólogos no fueron incorporados como técnicos de apoyo en una institución pública y el equipo de trabajo se desintegró. Por lo tanto, en el periodo de ejecución del proyecto, no se logró el reconocimiento formal del parabiólogo como un técnico local capacitado ni se logró su inserción en organismos locales públicos para la continuación de sus funciones.

Con el objetivo de formalizar la capacidad del parabiólogo como técnico reconocido por el ministerio de educación de Bolivia, desarrollamos el primer curso intensivo para la formalización de parabiólogos luego de 12 años de trabajo de campo y formación por la CABI y WCS. Este curso fue financiado por el Fondo Whitley para la Naturaleza, a través de un premio donado por Sting y Trudie Styler, y entregado a Erika Cuéllar en 2007. Como resultado del entrenamiento formal, 17 parabiólogos originarios (Isoseños, Chiquitanos, y Ayoreodes) del Chaco boliviano, luego de 8 meses con más de 800 horas de instrucción estructuradas en 13 módulos, proveídos por 20 instructores, obtuvieron sus certificados. Los módulos fueron los siguientes: Diseño de investigaciones de campo; Biología, ecología y taxonomía; Introducción a la ornitología; Mastozoología; Introducción a la herpetología; Entomología; Introducción a la ictiología; Introducción a la ecología botánica; Uso de GPS y aplicación básica de SIG; Introducción a la medicina veterinaria y su aplicación con la fauna silvestre; Introducción a las matemáticas; Análisis descriptivo, presentación y difusión y Primeros auxilios.

Además el curso incluyó 30 horas de sobrevuelos realizando censos aéreos en el área de distribución de la población del guanaco chaqueño Lama guanicoe. 
La experiencia de formación e inclusión de parabiólogos en un proyecto exitoso de investigación/conservación/manejo podría ser la base para la expansión de esta experiencia en otras áreas con cualidades biológicas y sociales similares. El objetivo de la participación activa de los parabiólogos es que las comunidades locales tomen la responsabilidad y hagan valer su autoridad para conservar la biodiversidad con un respaldo técnico apropiado. Por otro lado, el proceso de conservación necesita sumar capacidades locales que contribuyan al complejo proceso de integración de actores para beneficiar la conservación de especies/sitios focales. La conservación a largo plazo depende de la información robusta sobre las especies/sitios focales; del mantenimiento del interés de los actores locales con la conservación; de que los usuarios de los recursos naturales sientan que la conservación es aliado en lugar de ser competencia y que se sientan representados en los procesos de toma de decisiones acerca del destino de las zonas "fuente" de recursos. En este sentido el aporte que los parabiólogos podrían hacer para nutrir las bases de datos globales y mantener el vínculo entre la comunidad y la conservación sería muy valioso.

Sin embargo, el problema principal es la sostenibilidad de la inserción de parabiologos en el sistema de investigación, conservación y monitoreo. Desafortunadamente, el éxito post entrenamiento de esta iniciativa depende de la combinación de muchos factores que no siempre se alinean, como ser el interés de las instituciones correspondientes en apoyar e invertir seriamente en este proceso a largo plazo. La capacitación sin la garantía de continuación es una inversión a corto plazo. Por lo tanto, lo que se busca con la capacitación y posterior inserción de parabiólogos es fortalecer el paso inicial de este proceso y arraigarlo en un programa específico de conservación, para luego encaminar el proceso hacia la transferencia de la responsabilidad a organismos locales como áreas protegidas, organizaciones comunitarias, municipios y gobiernos departamentales en todo el país.

\section{Conclusiones}

El proceso histórico y situación actual del Pueblo Guaraní-Isoceño es especial y muy relevante para los otros Pueblos Indígenas de las Tierras Bajas de Bolivia. El Parque Nacional Kaa-lya fue en su momento la única área protegida en las Américas establecida como el resultado de las iniciativas de una organización indígena y la única que tuvo la responsabilidad administrativa primaria de la misma (Winer 2003). Esta aproximación, basada en principios de conservación y manejo sostenible de los recursos naturales contrastante a otros casos en Bolivia, donde las demandas por territorio para grupos indígenas y para áreas protegidas se superponían y eran fuente de conflictos basados en formas de competencia por la tenencia de tierra - dio a CABI la oportunidad de manejar $53.000 \mathrm{~km}^{2}$ en el Chaco Boliviano. Adicionalmente, CABI ha logrado articular consistentemente la superposición de jurisdicciones administrativas y de gestión ambiental y social, de acuerdo con disposiciones legales vigentes. En el mismo espacio están la Tierra Comunitaria de Origen (TCO) Isoso; el Distrito Municipal Indígena Isoceño; y el Área Protegida (Winer 2003).

Es evidente que los logros como producto de la colaboración de diferentes actores han sobrepasado enormemente la importancia de los compromisos que cada organización ha hecho en promover sus visiones y misiones respectivas. Esta perspectiva ha sido ratificada 
por una serie de reconocimientos internacionales importantes que han incrementado los incentivos para que sigan colaborando. Logros compartidos han contribuido a la confianza que cada uno tiene con el otro, y la voluntad de cada uno de hacer el esfuerzo adicional para buscar ese terreno compartido, basado en el reconocimiento de que cada institución logrará mucho más en cuanto el avance de sus respectivos objetivos a largo plazo a través de la colaboración, y no trabajando individualmente (Arellano 2003; Arambiza y Painter 2006; Castillo et al. 2006; Redford y Painter 2006; Castillo et al. 2007; Painter 2009; Painter et al. 2011).

Si bien la capacitación de parabiólogos fue enfocada en temas de biología metodológicos y analíticos, el contexto del entrenamiento fue basado en el respeto a sus capacidades, su cultura e intereses con relación a su medio ambiente. La metodología de trabajo con parabiólogos fue la del trabajo en equipo, promoviendo un aprendizaje mutuo. Complementario a esto último, durante las clases dentro del curso formal se promovió la discusión en grupos de culturas mixtas y las aptitudes individuales. Los parabiólogos fueron capacitados en el desarrollo de diseños de proyectos de investigación; en la colecta, el análisis e interpretación de datos; en la presentación de los resultados de sus investigaciones en diferentes versiones; y en la aplicación de los resultados en los procesos de manejo de sus recursos naturales. De hecho, algunos guardaparques han sido parabiólogos o actualmente tienen el liderazgo, ejecutando proyectos de desarrollo en sus comunidades. La combinación del conocimiento nato sobre historia natural, la pertenencia a una sociedad que utiliza los recursos naturales en forma directa, y el apoyo de la misma al desarrollo de esta formación técnica, hace de los parabiólogos pilares insustituibles en el proceso de apropiación de herramientas de conservación y manejo de recursos naturales.

El legado de la cooperación entre WCS y la CABI fue la formación de bolivianos y extranjeros en el estudio de los recursos naturales y su conservación. Actualmente varios de los parabiólogos formados durante esta alianza trabajan como guardaparques y los otros son líderes en sus comunidades ejecutando proyectos de desarrollo comunal.

Además, el plan de manejo elaborado durante este periodo de colaboración es la base del plan de manejo comunitario que está siendo llevado a cabo por técnicos de la $\mathrm{CABI}$, luego de ganar un financiamiento del Programa de Naciones Unidas para el Desarrollo (PNUD) para dicho propósito.

Los resultados obtenidos con relación a los mamíferos del Chaco han profundizado el conocimiento sobre: la historia natural, la situación de especies amenazadas e importantes para el consumo local, la distribución y densidad de algunas de las especies de mamíferos y la recuperación del hábitat para dichas especies. Esto último ha sido la base para generar propuestas de conservación y monitoreo a largo plazo, actualmente llevado a cabo por las mismas comunidades bajo la coordinación de la CABI.

Si bien es cierto que las bases de datos se nutrieron con el trabajo de tantos años de los que formamos parte de esta alianza, los beneficios a largo plazo van más allá de los informes técnicos, las publicaciones científicas o el mejor conocimiento de nuestra vida silvestre. Nos referimos a que la contribución mayor de esta alianza es el empuje de actores locales, concretando su visión y prioridades, para continuar con un proyecto mayor de conservación e integración a largo plazo. 
A J. Ayala, H. Azurduy, Z. Barahona, O. Castillo, R. Leny Cuéllar, S. Deem, P. Feinsinger, C. Fiorello, J. Guerrero, A. Hesse, T. Hibbitts,P. Hutchison, A. Jahn, L. Maffei, A. M. Mamani, R. Miserendino, R. Montaño, G. Navarro, H. Noss, M. Painter, C. Pinto, P. Rebolledo, A. Romero-Muñoz, D. Rumiz y L. Siles. Este trabajo se realizó como parte del programa de WCS-Bolivia para la conservación del paisaje Kaa-lya. En parte se realizó gracias al apoyo de la United States Agency for International Development (USAID/Bolivia Cooperative Agreement No. 511-A-00-01-00005). Las opiniones expresadas representan a los autores y no necesariamente reflejan los criterios de USAID. Agradecemos a la Capitanía del Alto y Bajo Isoso y a la Dirección General de Biodiversidad por autorizar y apoyar el programa. Agradecemos a las autoridades de Isoso, comunarios, cazadores, parabiólogos y monitores de cacería de Isoso por su apoyo y participación permanente.

\section{Literatura citada}

Aguirre, L. F., R. Aguayo, J. Balderrama, C. Cortéz, y T. Tarifa (eds.). 2009. Libro Rojo de la fauna silvestre de vertebrados de Bolivia. Ministerio de Medio Ambiente y Agua de Bolivia. La Paz, Bolivia.

Alıaga-Rossel, E., A. J. Noss, y L. Maffel. 2009. Catagonus wagneri (Rusconi, 1930): Artiodactyla - Tayassuidae. Pp. 477-478 en Libro Rojo de la fauna silvestre de vertebrados de Bolivia (Aguirre, L. F., R. Aguayo, J. Balderrama, C. Cortéz, y T. Tarifa, eds.). Ministerio de Medio Ambiente y Agua. La Paz, Bolivia.

Altrichter, M., A. Taber, A. J. Noss, y L. Maffel. 2008. Catagonus wagneri. Lista roja de especies amenazadas. UICN.

Arambiza, E., y M. Painter. 2006. Biodiversity conservation and the quality of life of indigenous people in the Bolivian Chaco. Human Organization 65:20-34.

Arellano, S. 2003. Bolivia: soluciones a conflictos de tierra. Estudio de caso: la Capitanía de Alto \& Bajo Izozog (CABI). Chemonics Internacional Inc. Washington, EE.UU.

Arispe, R., E. Cuéllar, y I. Combès. 2005. Jaguares y pumas, el problema de la depredación del ganado: guía informativa. Wildlife Conservation Society. Santa Cruz de la Sierra, Bolivia.

Arispe, R., D. I. Rumiz, C. Venegas, y A. J. Noss. 2009. El conflicto de la depredación de ganado por el jaguar Panthera onca en Santa Cruz, Bolivia. Manejo de Fauna en Latinoamérica. Pp. 1-23

Banegas, J., y L. Maffel. 2007. Estudio del pecarí del Chaco o solitario Catagonus wagneri en el Parque Nacional Kaa-lya. Wildlife Conservation Society. Santa Cruz de la Sierra, Bolivia.

Barrientos, J., y R. L. Cuéllar. 2009. Iniciativas de manejo de fauna silvestre mediante reglamentos de cacería en las comunidades de Isoso del Gran Chaco. Revista Electrónica Manejo de Fauna Silvestre en Latinoamérica 2: Pp.1-9

Baumgartner, L. L., y A. C. Martin. 1939. Plant histology as an aid in squirrel food-habit studies. Journal of Wildlife Management 3:266-268.

Borchers, D. L., y M. G. EFFord. 2008. Spatially explicit maximum likelihood methods for capture-recapture studies. Biometrics 64:377-385. 
Castillo, O., Z. Barahona, D. Mendía, A. Noss, A. Hesse, A. Burbano, y J. C. Chivé. 2007. Sistemas de gestión integrada \& gobernanza en Áreas Protegidas de Bolivia, Ecuador \& Perú: desde la visión de sus comités de gestión. p. 280. WCS \& Tinker Foundation. Santa Cruz de la Sierra.

Castillo, O., C. Clark, P. Coppolillo, H. Kretser, R. McNab, A. Noss, H. Quieroz, Y. Tessema, A. Vedder, R. Wallace, J. Walston, y D. Wilkie. 2006. Casting for Conservation actors: people, partnerships and wildlife. Working paper No. 28. Wildlife Conservation Society. New York, EE.UU.

Cú́llar, E. 2000a. Automonitoreo de la cacería de armadillos en el Izozog, Gran Chaco Boliviano. Pp. 113-118 en Manejo de fauna silvestre en Amazonía y Latinoamérica (Cabrera, E., C. Mercolli, y R. Resquin, eds.). CITES Paraguay, Fundación Moises Bertoni, University of Florida. Asunción, Paraguay.

Cú́llar, E. 2011. Ecology and conservation of the guanaco Lama guanicoe in the Bolivian Chaco: habitat selection within a vegetation succession. PhD Dissertation. University of Oxford. Oxford, EE.UU.

Cuéllar, E., y I. Combès. 2002. Jaguares, pumas y ganado: guía informativa. Wildlife Conservation Society. Santa Cruz de la Sierra, Bolivia.

Cuéltar, E., y A. Noss. 1997. Conteo de huellas en brechas barridas: un índice de abundancia para mamíferos. Ecología en Bolivia 30:55-67.

Cuéllar, E., y A. J. Noss. 2003. Mamíferos del Chaco y de la Chiquitanía. Editorial FAN. Santa Cruz de la Sierra, Bolivia.

Cú́llar, E., y A. Núñez. 2009. Lama guanicoe (Müller, 1776 ): Artiodactyla-Camelidae. Pp. 454-456 en Libro Rojo de la fauna silvestre de vertebrados de Bolivia (Aguirre, L. F., R. Aguayo, J. Balderrama, C. Cortéz, y T. Tarifa, eds.). Ministerio de Medio Ambiente y Agua de Bolivia. La Paz, Bolivia.

Cuéllar, E., J. Segundo, G. Castro, A. Segundo, y A. Noss. 2005. Current status of the Guanaco Lama guanicoe voglii in the Bolivian Chaco. Annual report to Shared Earth Foundation and Wildlife Conservation Society. WCS-Bolivia, Santa Cruz.

Cú́llar, R. L. 2000b. Uso de los animales silvestres por pobladores Izoceños. Pp. 471-484 en Manejo de fauna silvestre en Amazonía y Latinoamérica (Cabrera, E., C. Mercolli, y R. Resquin, eds.). CITES Paraguay, Fundación Moises Bertoni, University of Florida. Asunción, Paraguay.

Cuéllar, R. L., D. Alarcón, F. Peña, C. Méndez, A. Romero-Muñoz, L. Maffei, D. I. Rumiz, y A. J. Noss. 2012. Kaaiyana: a jaguar with cubs in the Kaa-lya del Gran Chaco National Park, Bolivia. Cat News 57: 4-6.

Cuéllar, R. L., y A. J. Noss. 2002. Plan de manejo para el uso comercial del taitetú Tayassu tajacu en el Izozog. Proyecto Kaa-lya, CABI y Wildlife Conservation Society. Santa Cruz de la Sierra, Bolivia.

Cuéllar, R. L., A. J. Noss, y A. Arambiza. 2004. El registro de la cacería como base para el monitoreo y manejo de fauna en Isoso. Revista Boliviana de Ecología y Conservación Ambiental 16:29-40.

EisenberG, J. F., y K. H. Redford. 1999. Mammals of Neotropics - the Central Neotropics: Ecuador, Peru, Bolivia, Brazil. University of Chicago Press. Chicago, EE.UU. 
Hansen, M. C., P. V. Potapov, R. Moore, M. Hancher, S. A. Turubanova, A. Tyukavina, D. Thau, S. V. Stehman, S. J. Goetz, T. R. Loveland, A. Kommareddy, A. Egorov, L. Chini, C. O. Justice, y J. R. G. Townshend. 2013. High-Resolution Global Maps of 21 stCentury Forest Cover Change. Science 342: 850-853.

Hesse, A. J., y E. Cuéllar. (eds.). 2008. Manuales técnicos, 1 er curso de capacitación para la formación de Parabiólogos. Whitley Fund for Nature and Wildlife Conservation Society. Santa Cruz de la Sierra, Bolivia.

JANZEN, D. H. 2002. Tropical dry forest: Area de Conservacion Guanacaste, northwestern Costa Rica. Handbook of Ecological Restoration 2:559-583.

Janzen, D. H. 2004. Setting up tropical biodiversity for conservation through nondamaging use: participation by parataxonomists. Journal of Applied Ecology 41:181-187.

MafFel, L. 2000. Estructura de edades de la urina Mazama gouazoubira en el Chaco Boliviano. Pp. 373-376 en Manejo de fauna silvestre en Amazonía y Latinoamérica (Cabrera, E., C. Mercolli, y R. Resquin, eds.). CITES Paraguay, Fundación Moises Bertoni, University of Florida. Asunción, Paraguay.

MAfFel, L. 2001. Estructura de edades de la urina Mazama gouazoubira en el Chaco boliviano. Mastozoología Neotropical 8:149-155.

Maffel, L. 2003. The age structure of tapirs Tapirus terrestris in the Chaco. Tapir Conservation 12:18-19.

Maffel, L. 2004. Age structure of two hunted peccary species in the Bolivian Chaco Tayassu tajacu and T. pecari. Mammalia 67:575-578.

Maffel, L., E. Cuéllar, y A. Noss. 2004. One thousand jaguars Panthera onca in Bolivia's Chaco? Camera trapping in the Kaa-lya National Park. Journal of Zoology 262:295304.

Morello, J., y G. Hortt. 1985. Changes in the area extent of arable farming, stock raising and forestry in the South American Chaco. Applay to Geographycal Development 25:109-127.

Morello, J., y C. Saravia-Toledo. 1959. The Chaco Argentina forest: cattle raising and forests in eastern Salta. Revista de Agronomia del Noroeste Argentino 3:209-258.

Navarro, G. 2002. Ecología estructural y dinámica de áreas quemadas en el PN-ANMI Kaa-lya del Gran Chaco. Pp. 17-90 en Ecología del fuego en el Parque Nacional y Área Natural de Manejo Integrado Kaa-lya del Gran Chaco (Guerrero, J., ed.). Proyecto Kaa-lya. Santa Cruz, Bolivia.

Navarro, G., y A. Fuentes. 1999. Geobotánica y sistemas ecológicos de paisaje en el Gran Chaco de Bolivia. Revista Boliviana de Ecología 5:25-50.

Noss, A. 1998. El monitoreo comunitario de cacería en el Izozog: datos preliminares. Ecología en Bolivia 31:53-66.

Noss, A. 1999. Manejo de fauna comunitario en el Gran Chaco. Pp. 109-116 en Manejo y conservación de fauna silvestre en América Latina (Fang, T. G., O. L. Montenegro, y R. E. Bodmer, eds.). Instituto de Ecología. La Paz, Bolivia.

Noss, A., E. Cuéllar, H. Gómez, T. Tarifa, y E. Aliaga-Rossel. 2010a. Dasypodidae. Pp. 173-212 en Distribución, ecología y conservación de los mamíferos medianos y grandes de Bolivia (Wallace, H. G. R. B., Z. R. Porcel, y D. I. Rumiz, eds.). Centro de Ecología Difusión Simón I. Patiño. Santa Cruz de la Sierra, Bolivia. 
Noss, A. J. 2000. La sostenibilidad de la cacería de subsistencia Izoceña. Pp. 535-544 en Manejo de fauna silvestre en Amazonía y Latinoamérica (Cabrera, E., C. Mercolli, y R. Resquin, eds.). CITES Paraguay, Fundación Moises Bertoni, University of Florida. Asunción, Paraguay.

Noss, A. J., y E. Cuétlar. 2000. Índices de abundancia para fauna terrestre en el Chaco boliviano: huellas en parcelas y en brechas barridas. Pp. 73-82 en Manejo de fauna silvestre en Amazonía y Latinoamérica (Cabrera, E., C. Mercolli y R. Resquin, eds.). CITES Paraguay, Fundación Moises Bertoni, University of Florida. Asunción, Paraguay.

Noss, A. J., E. Cuéllar, y R. L. Cuellar. 2004a. An evaluation of hunter self-monitoring in the Bolivian Chaco. Human Ecology 32:685-702.

Noss, A. J., E. Cuéllar, y R. L. Cuéllar. 2003a. Hunter self-monitoring as a basis for biological research: data from the Bolivian Chaco. Mastozoología Neotropical 10:49-67.

Noss, A. J., y R. L. Cuellar. 2008. The sustainability of hunting Tapirus terrestris and Tayassu pecari in the Isoso indigenous communal land: the unified harvest model. Mastozoologia Neotropical 15:241-252.

Noss, A. J., y R. L. Cuéllar. 2001. Community attitudes towards wildlife management in the Bolivian Chaco. Oryx 35:292-300.

Noss, A. J., R. L. Cuéllar, A. Arambiza, y J. Barrientos. 2010b. Sostenibilidad de la cacería en el Chaco: 12 años de manejo de fauna silvestre en la Tierra Comunitaria de Orígen Isoso. Pp. 1-36 en Experiencias de manejo de fauna silvestre en Bolivia (Gómez, H., y A. Llobet, eds.). Fundacion Amigos de la Naturaleza. Santa Cruz de la Sierra, Bolivia.

Noss, A. J., R. L. Cuellar, y J. Ayala. 2006. Drive counts for grey brocket deer in the Bolivian Chaco. Mammalia 70:64-69.

Noss, A. J., R. L. Cuéllar, y E. Cuéllar. 2008. Exploitation of xenarthrans by the GuaraníIsoseño indigenous people of the Bolivian Chaco: comparisons with hunting by other indigenous groups in Latin America, and implications for conservation. Pp. 244-254 en The biology of the Xenarthra (Vizcaino, S. F., y W. J. Loughry, eds.). University of Florida Press. Gainesville, Florida.

Noss, A. J., J. Guerrero, R. L. Cuellar, y Z. Barahona. 2003b. El uso comercial de recursos naturales mediante el manejo comunitario: la experiencia del pueblo izoceño en Bolivia. Pp. 287-314 en Fauna socializada: tendencias en el manejo participativo de la fauna en América Latina (Campos-Rozo, C., y A. Ulloa, eds.). Fundación Natura. Bogotá, Colombia.

Noss, A. J., M. A. Mares, y M. M. Díaz. 2002. The Chaco. Pp. 164-173 en Wilderness: earth's last wild places (Mittermeier, R. A., C. G. Mittermeier, P. R. Gil, J. Pilgrim, G. Fonseca, T. Brooks, y W. R. Konstant, eds.). CEMEX, Conservation International, and Sierra Madre. Washington, EE.UU.

Noss, A. J., I. Oetting, y R. L. Cuéllar. 2005. Hunter self-monitoring by the IsoseñoGuaraní in the Bolivian Chaco. Biodiversity and Conservation 14:2679-2693.

Noss, A. J., R. Peña, y D. I. Rumiz. 2004b. Camera trapping Priodontes maximus in the dry forests of Santa Cruz, Bolivia. Endangered Species Update 21:43-52. 
Olds, N., S. Anderson, y T. L. Yates. 1987. Notes on Bolivian mammals 3. A revised diagnosis of Andalgalomys (Rodentia, Muridae) and the Description of a New Subspecies. American Museum Novitates: 1-17.

Painter, M. 2009. Rights-based conservation and the quality of life of indigenous people in the Bolivian Chaco. Pp. 163-184 en Rights-based approaches, exploring issues and opportunities for conservation (Campese, J., T. Sunderland, T. Greiber, y G. Oviedo, eds.). IUCN \& CIFOR. Bogor, Indonesia.

Painter, R. L. E., A. Duran, y E. Miro. 2011 . Indigenous Alliances for Conservation in Bolivia. Conservation Biology 25:1084-1086.

Pimbert, M. P., y J. N. Pretty (eds.). 1994. Participation, People and the Management of National Parks and Protected Areas: Past Failures and Future Promise. Ginebra, Suiza.

Pinto, C. 2005. Análisis multitemporal de cambios de vegetación de la pampa chaqueña, en la zona de los arenales de Yanahigua, oeste del Parque Nacional Kaa-lya. Technical report 87. Proyecto Kaa-lya. Santa Cruz, Bolivia.

Redford, K. H., y E. Fearn. 2007. Protected areas and human displacement: a conservation perspective. Working paper No. 29. Wildlife Conservation Society. New York, EE.UU.

Redford, K. H., y M. Painter. 2006. Natural alliances between conservationists and indigenous peoples. Technical Report 25. Wildlife Conservation Society. New York, EE.UU.

Redford, K. H., A. Taber, y J. A. Simonetti. 1990. There is more to biodiversity than the tropical rain-forests. Conservation Biology 4:328-330.

Rojas-Suárez, C. R., y L. Maffel. 2004. Estructura de edades de la hurina Mazama guazoubira, taitetú Tayassu tajacu, tropero Tayassu pecari y anta Tapirus terrestris. Pp. 261-264 en Manejo de Fauna Silvestre en la Amazonía y Latinoamérica. www. revistafauna.com.pe/memo.htm. Iquitos, Perú.

Romero-Muñoz, A., L. Maffei, E. Cuéllar, y A. J. Noss. 2010. Temporal separation between jaguar and puma in the dry forests of southern Bolivia. Journal of Tropical Ecology 26:303-311.

Romero-Muñoz, A., A. J. Noss, L. Maffel, y R. R. Montaño. 2007. Binational population of jaguars confirmed by camera-trapping in the American Gran Chaco. Cat News 46:24-25.

Rumiz, D. 2004. Elementos básicos para la preparación y evaluación de planes de manejo de fauna. Revista Boliviana de Ecología y Conservación Ambiental 16:99104.

SChofield, C. J., y E. H. BuCher. 1986. Industrial contributions to desertification in South America. Trends in Ecology and Evolution 1:78-80.

Segundo, J. 2007. Conociendo la población de guanacos en el Chaco Boliviano. Pp. 22. Wildlife Conservation Society. Santa Cruz de la Sierra, Bolivia.

Silver, S. 2004. Assessing jaguar abundance using remotely triggered cameras. Wildlife Conservation Society.

Taber, A., G. Navarro, y M. A. Arribas. 1997. A new park in the Bolivian Gran Chaco - An advance in tropical dry forest conservation and community-based management. Oryx 31:189-198. 
TABer, A. B. 1991. The status and conservation of the Chacoan peccary in Paraguay. Oryx 25:147-155.

TARIFA, T. 2009. Priodontes maximus (Kerr, 1792): Cingulata - Dasypodidae. Pp. 496498 en Libro Rojo de la fauna silvestre de vertebrados de Bolivia (Aguirre, L. F., R. Aguayo, J. Balderrama, C. Cortéz, y T. Tarifa, eds.). Ministerio de Medio Ambiente y Agua, Bolivia, La Paz, Bolivia.

UICN. 2010. Lista Roja de Especies Amenazadas. www.iucnredlist.org.

Villalba, L. 2004. Monitoreo de la población de Lama guanicoe vog/ii. DesdelChaco Foundation. Loma Plata, Paraguay.

Wetzel, R. M., R. E. Dubois, R. L. Martin, y P. Myers. 1975. Catagonus, an 'extinct' peccary, alive in Paraguay. Science 189:379-381.

Wiluiams, O. B. 1962. A technique for studying microtine food habits. Journal of Mammalogy 43:365-368.

Winer, N. 2003. Review and strategic planning consultancy Kaa-lya del Gran Chaco project. Wildlife Conservation Society. Santa Cruz, Bolivia.

Sometido: 21 de enero de 2014

Revisado: 10 de abril de 2014

Aceptado: 14 de abril de 2014

Editor asociado: Robert Owen

Diseño gráfico editorial: Gerardo Hernández 